\title{
Ab Initio Study of Functionalized Carbon Nanotubes
}

\author{
K. Milowska, M. Birowska And J.A. Majewski \\ Institute of Theoretical Physics, Faculty of Physics, University of Warsaw, Hoża 69, 00-681 Warszawa, Poland \\ In the present paper, we study the stability of $(9,0),(10,0),(11,0)$ carbon nanotubes functionalized \\ with simple organic molecules $-\mathrm{CH}_{n}$ (for $n=2,3,4$ ). Our studies are based on ab initio calculations within \\ the framework of the density functional theory. We determine binding energies of the functionalized carbon \\ nanotubes and the changes in the geometry and electronic structure caused by the functionalization. We \\ observe the characteristic effects such as rehybridization of the bonds induced by fragments attached to carbon \\ nanotubes and pentagon/heptagon $(5 / 7)$ defects in $-\mathrm{CH}_{2}$ functionalized carbon nanotubes. We study also \\ dependence of the binding energies of the functionalized carbon nanotubes on the density of the adsorbed \\ molecules and diameter of the single-wall carbon nanotubes. Our calculations reveal that the $-\mathrm{CH}_{2}$ fragments \\ exhibit the strongest cohesion and we determine the critical density of the $-\mathrm{CH}_{2}$ fragments which could be adsorbed.
}

PACS numbers: 31.15.ae, 31.15.E-, 61.46.Fg, 61.48.De, 73.22.-f, 81.07.De

\section{Introduction}

Recently functionalized carbon nanotubes (CNTs) attract a lot of research activity, mostly because of their potential applications as electrical sensors of chemical or biological substances and also as essential constituents of composite materials. In composite materials, only simple organic molecules (such as $-\mathrm{CH}_{n}$ or $-\mathrm{NH}_{n}$ fragments) adsorbed at the surface of CNTs allow for strong binding of the functionalized in such a way CNTs with matrix material, typically a polymer or a metal [1-4]. On the other hand, the process of adhesion of molecules to the walls of CNTs leads to changes of the electronic and mechanical properties of the CNTs. One of the most important issues is the problem of the stability of the functionalized CNTs. In particular, establishing the critical density of the molecules that can be adsorbed at the surface of the CNTs without causing major damage is of great practical importance, since it facilitates engineering of nanocomposites. Some aspects of the cohesion in functionalized CNTs have been studied previously $[1,5-8]$. We have performed extensive studies of the stability of the functionalized CNTs with various molecular groups. In this letter, we focus on the single wall CNTs (SWNTs) of various diameters functionalized with the $-\mathrm{CH}_{n}$ fragments and report the results of ab initio studies of their stability.

\section{Method}

Our studies of the CNTs functionalized with $\mathrm{CH}_{n}$ fragments are based on the $a b$ initio calculations in the framework of the Kohn-Sham realization of the density functional theory (DFT) $[9,10]$. We have used the generalized gradient approximation (GGA) for the exchange correlation density functional [11] and supercell geometry with periodic boundary conditions within the numerical package SIESTA. A kinetic energy cut-off of 150 Ry and split double zeta basis set without spin polarization have been used in all calculations. The lateral separation (i.e., lateral lattice constants in $x$ and $y$ direction) has been set to $20 \AA$, just to eliminate completely the spurious interaction between neighboring cells. Along the $z$ direction (i.e., along the axis of the tube), the natural lattice constant resulting from the chiral numbers $n$ and $m$ has been chosen. The positions of all atoms in the supercell have been optimized to get vanishing forces on atoms. Hereafter, we present the results of the calculations for the $(9,0),(10,0),(11,0)$ SWNTs functionalized with $\mathrm{CH}_{n}$ (for $n=2,3,4$ ) fragments, which has been attached on the sidewalls of CNTs.

\section{Results and discussion}

The binding energies of the functionalized CNTs, i.e., the difference of the total energy of the functionalized CNTs and the total energy of the all free constituent atoms, provide a measure of the stability of the functionalized CNTs. In Fig. 1, the binding energies of the $(9,0)$ SWNTs functionalized with $-\mathrm{CH}_{2},-\mathrm{CH}_{3}$, and $\mathrm{CH}_{4}$ are depicted for various density of functionalizing fragments. It is clearly seen that the strongest cohesion of the fragments to the $\mathrm{CNT}$ occurs in the case of $-\mathrm{CH}_{2}$ fragments and that the binding energy generally decreases with the density of the fragments. In the case of $-\mathrm{CH}_{3}$ group the binding becomes extremely weak for higher densities. In the case of $\mathrm{CH}_{4}$ molecules, we observe the dissociation of the $\mathrm{CH}_{4}$ into $-\mathrm{CH}_{2}$ and $\mathrm{H}_{2}$ dimer; $-\mathrm{CH}_{2}$ part binds to the CNT (but weaker than the $\mathrm{CH}_{2}$ group alone) and $\mathrm{H}_{2}$ dimer remains unbound.

The characteristic geometrical features of the $(9,0)$ SWNT functionalized with $\mathrm{CH}_{n}$ groups are depicted in 


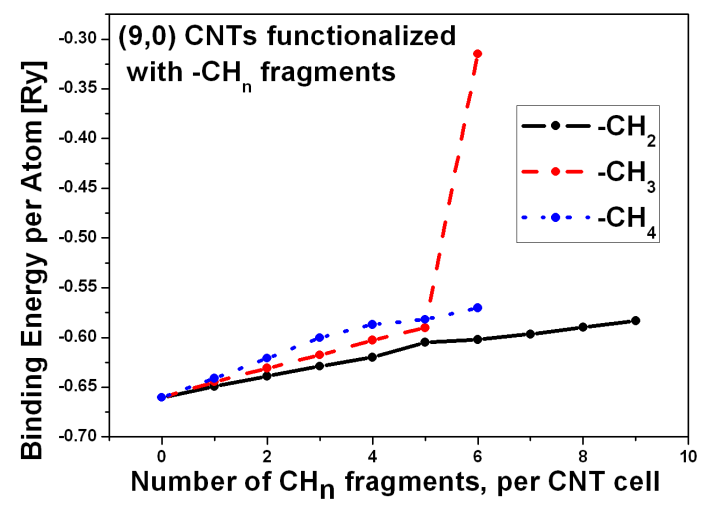

Fig. 1. Binding energies of functionalized $(9,0)$ CNT for various $\mathrm{CH}_{n}$ fragments and their densities measured as the number of groups per CNT cell.
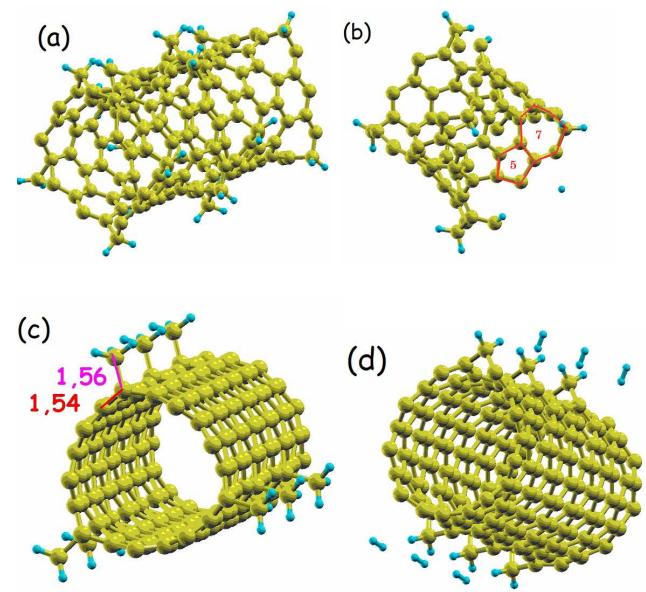

Fig. 2. Geometry of the $(9,0)$ SWNT functionalized by various fragments. (a), (b) $-\mathrm{CH}_{2}$ fragments - strong deformation of the SWNT in form of characteristic pentagons and heptagons is depicted; (c) $-\mathrm{CH}_{3}$ group change of the type of hybridization from $s p^{2}$ (graphene) to $s p^{3}$ (diamond), the rather large lengths of $\mathrm{C}-\mathrm{C}$ bonds are indicated [in $\AA$ ]; (d) $\mathrm{CH}_{4}$ molecule - dissociation into $-\mathrm{CH}_{2}$ fragment bound to the tube and $\mathrm{H}_{2}$ unbound dimer.

Fig. 2. Our calculations reveal also the physical mechanisms of the functionalization. Generally, the functionalization of CNTs with $-\mathrm{CH}_{n}$ groups causes large deformations of the CNTs. In particular, we have observed characteristic effect of pentagon/heptagon (5/7) defects in CNTs functionalized with $-\mathrm{CH}_{2}$ fragments (see Fig. 2b), which has been previously observed in literature during the plastic yielding CNTs [1]. $-\mathrm{CH}_{3}$ groups (Fig. 2c) induce local distortions along the radial direction on the tube side wall. It can be understood by the local $s p^{3}$ rehybridization of the carbon-carbon type of bonding. The bond lengths between carbon atoms from the CNT and the group and between $\mathrm{C}$ atoms in the hexagonal ring of the CNT, are close to the bond length in the $s p^{3}$-hybridized diamond $(1.54 \AA)$ and are significantly larger than the $\mathrm{C}-\mathrm{C}$ bond length in the perfect graphene sheet $(1.42 \AA)$ with the $s p^{2}$ hybridization.

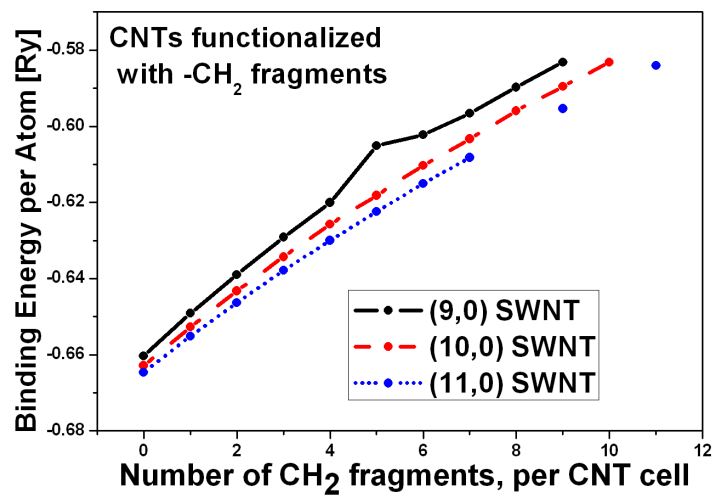

Fig. 3. Binding energy of the $-\mathrm{CH}_{2}$ functionalized CNTs as a function of the group density for various diameter of the SWNTs.

We have also addressed the important problem how the stability of the functionalized CNTs depends on its diameter. In Fig. 3 the binding energies for the $-\mathrm{CH}_{2}$ functionalized $(9,0),(10,0),(11,0)$ SWNTs are depicted. It can be seen that the binding energy exhibits weak dependence on the diameter of the CNTs with the tendency of stronger cohesion in the case of CNTs of larger diameter. On the basis of our calculations, we are also able to predict the critical density of the adsorbed groups, which is equal to ten $-\mathrm{CH}_{2}$ fragments per unit cell of the $(11,0)$ SWNT. Attachment of more than ten groups causes irreversible damage to the CNTs. It is important issue concerning the design of composite materials utilizing the CNT enforcement. The studies of the elastic properties of the functionalized CNTs are under way and will be published elsewhere.

\section{Conclusions}

We have performed $a b$ initio studies of stability of CNTs functionalized with $-\mathrm{CH}_{n}$ fragments. It turns out that the physical mechanism of the functionalization can be understood in terms of rehybridization of the bonds induced by adsorbed group. We predict that the critical density of the attached $-\mathrm{CH}_{2}$ fragments is equal to ten fragments per CNT cell.

\section{Acknowledgments}

The authors gratefully acknowledge financial support of the Polish Council for Science through the Development Grants for the years 2008-2011 (No. 15-0011$-04 / 2008$, No. KB/72/13447/IT1-B/U/08).

\section{References}

[1] W.A. Goddard III, D.W. Brenner, S.E. Lyshevski, G.J. Iafrate, Handbook of Nanoscience, Engineering, and Technology (Electrical Engineering Handbook), CRC Press LLC, Boca Raton, FL, USA 2003. 
[2] J. Gou, Z. Liang, C. Zhang, B. Wang, Composites: Part B 36, 524 (2005).

[3] D. Srivastava, C. Wei, K. Cho, Appl. Mech. Rev. 56, 215 (2003).

[4] C. Wei, D. Srivastava, K. Cho, Nanoletters 4, 1949 (2004).

[5] M. Rosi, Ch.W. Bauschlicher Jr., Chem. Phys. Lett. 437, 99 (2007).

[6] B.K. Agrawal, S. Agrawal, S. Singh, R. Srivastava, J. Phys., Condens. Matter 18, 4649 (2006).
[7] F. Li, Y. Xia, M. Zhao, X. Liu, B. Huang, Z. Tan, Y. Ji, Phys. Rev. B 69, 165415 (2004).

[8] M.V. Veloso, A.G. Souza Filho, J. Mendes Filho, S.B. Fagan, R. Mota, Chem. Phys. Lett. 430, 71 (2006).

[9] P. Hohenberg, W. Kohn, Phys. Rev. 136, 864 (1964).

[10] W. Kohn, L.J. Sham, Phys. Rev. 140, A1133 (1965).

[11] J.P. Perdew, K. Burke, M. Ernzerhof, Phys. Rev. Lett. 77, 3865 (1996). 